\title{
Essays on James Clarence Mangan
}

\section{Claude Fierobe}

\section{OpenEdition}

12 Journals

\section{Édition électronique}

URL : http://journals.openedition.org/etudesirlandaises/4750

DOI : 10.4000/etudesirlandaises.4750

ISSN : 2259-8863

\section{Éditeur}

Presses universitaires de Caen

\section{Édition imprimée}

Date de publication : 15 décembre 2015

Pagination : 159-160

ISBN : 978-2-7535-4366-9

ISSN : 0183-973X

\section{Référence électronique}

Claude Fierobe, «Essays on James Clarence Mangan », Études irlandaises [En ligne], 40-2 | 2015, mis en ligne le 15 décembre 2015, consulté le 24 septembre 2020. URL : http://journals.openedition.org/ etudesirlandaises/4750; DOI : https://doi.org/10.4000/etudesirlandaises.4750

\section{(c) (i) (9)(2)}

Études irlandaises est mise à disposition selon les termes de la Licence Creative Commons Attribution - Pas d'Utilisation Commerciale - Partage dans les Mêmes Conditions 4.0 International. 
égard à la thématique générale de l'ouvrage, une plus large représentativité de chercheurs d'Europe continentale eut été souhaitable. L'échange de points de vue en eut été d'autant enrichi.

De la même façon, outre Kate O'Brien dont l'œuvre fait l'objet d'un chapitre, tous les écrivains étudiés ici sont des hommes. Il eut été bienvenu à cet égard, et pertinent sur le plan scientifique, d'inclure un chapitre sur la production littéraire d'Elizabeth Bowen, écrivaine irlandaise qui s'impliqua si activement lors du conflit qu'elle fut soupçonnée d'espionnage. Bowen est évoquée à plusieurs reprises et citée dans la préface, mais de façon insuffisante. Or, la présence d'un article sur son œuvre eut été tout à fait justifiée dans un ouvrage comme celui-ci.

Indépendamment de ces réserves, cette étude est passionnante et d'une lecture très agréable. Elle permet de revisiter une décennie culturellement riche et, selon les termes de R. F. Foster, de "découvrir un territoire imaginaire resté trop longtemps inexploré ».

Bertrand CARDIN

Université de Caen Normandie

Sinéad Sturgeon (ed.), Essays on James Clarence Mangan, The Man in the Cloak, Basingstoke, Palgrave Macmillan, 2014, XVI + 242 p., ISBN 9781137273376.

Au début un poème de Paul Muldoon "A Night on the Tiles With J. C. Mangan " suivi d'une préface tonique de J. Chuto - Mangan n'est pas seulement the "Nameless One " mais aussi the " Dispossessed One " - à la fin une passionnante postface où Ciaran Carson, évoque la "ghostly presence " de Mangan qui hante son roman Exchange Place (2012). Dans ce cadre, dix essais de haute tenue, sans exception, qui balaient et balisent une ouvre protéiforme. Pour David Lloyd la traduction d'un poème de Uhland ("Auf der Überfahrt ", devenu "Spirits Everywhere ") est à la fois " a form of possession or necromancy", et une réflexion sur le travail du traducteur. Sur le même sujet, David Wheatley montre le danger de priver de sa complexité une ouvre plus centrifuge que centripète qui échappe au schéma binaire "Irish/English ", et fait émerger "the polyglot energies dormant in any language ". "Night Singer : Mangan Among the Birds" (S. Sturgeon) : romantisme et rossignol, mais aussi perroquet et corbeau, tous deux images du traducteur, le premier se contentant de répéter mais à sa manière bien à lui, le second pillant le bien d'autrui. Dans les "psychological narratives ", Richard Haslam explore les modes du surnaturel, "the phantasmagoria of the mind ", à travers le prisme irlandais de l'ironie romantique. Souvent décrit comme "existing 
in, or haunted by, ancient Ireland ", Mangan doit, selon Joseph Lennon, être situé dans le contexte culturel plus vaste d'une véritable vision, "distinguishing an Irish past to create an Irish future ". Allant encore plus loin, Cóilin Parsons écoute une voix qui, à l'instar de Goethe et de la Weltliteratur, certes avec des différences, vise à saisir la totalité du monde "in all its glorious and gruesome reality ". Dans Ulysses Stephen Dedalus est peint "in the gothic shadow of Mangan's restless ghost ", écrit John McCourt : Mangan est un "internal exile », à la fois emblème et victime de son pays, écrivain si novateur que même Joyce "struggled to keep up with him". $\mathrm{Au}$ sein des débats contemporains sur les pouvoirs respectifs de la science et du spiritualisme, Anne Jamison étudie la "spiritualist vastation " d'un homme fasciné par figure du magicien et par le surnaturel, pour qui la réalité n'est sans doute qu'une illusion. Vu la complexité déroutante d'une ouvre qui échappe à toute orthodoxie, Sean Ryder ("Unauthorized Mangan ») conclut : "Every edition is a construct... The fantasy of getting back to the original is absurd in a fundamental sense" : à chacun son Mangan. Dans "Mangan in England ", Matthew Campbell dresse un parallèle convaincant entre Browning et Mangan, "writing a contorted art in terrible times ", pour révéler, dans le contexte victorien, le sens profond de la marginalité d'un poète qui, sous le masque de «Selber » dans « Twenty Golden Years ago ", se disait "tortured torturer of reluctant rhymes".

Rappelant les acquis et ouvrant en même temps de nouvelles perspectives, ce recueil d'essais fait honneur à Sinéad Sturgeon et aux auteur(e)s. Bibliographie, index et notes le complètent pour constituer un remarquable outil de travail. Sur la couverture d'une élégante reliure, verte bien sûr, se détache l'énigmatique silhouette noire de "l'homme à la cape ", comme une invitation à s'aventurer plus loin, en sa compagnie, dans un territoire où beaucoup reste à découvrir.

Claude Fierobe

Université de Reims Champagne-Ardenne

Terri Hooley, Richard Sullivan, Hooleygan: Music, Mayhem and Good Vibrations, Belfast, Blackstaff, 2010, 234 p. ISBN 9780856408519

First published in 2010, two years before the release of the film Good Vibrations film which was based on Terry Hooley's life and which renewed interest in Northern Ireland's 1970s punk scene, Hooleygan: Music, Mayhem, Good Vibrations is a biography written by journalist Richard Sullivan and is based on his conversations with Terri Hooley and his entourage. The book begins with an account of how Hooley acquired his glass eye: when he was six years old, a stray toy arrow 